\title{
Symmetries of the generalized Calogero model and the Polychronakos-Frahm chain
}

\author{
Tigran Hakobyan \\ Yerevan State University, 1 Alex Manoogian St., Yerevan 0025, Armenia
}

(Received 28 March 2019; published 21 May 2019)

\begin{abstract}
The symmetry of the generalized Polychronakos-Frahm chain is obtained from the Dunkl-operator deformation of the unitary algebra, which describes the symmetry of the generalized Calogero model.
\end{abstract}

DOI: 10.1103/PhysRevD.99.105011

\section{INTRODUCTION}

Integrable chains with inverse-square interactions were first introduced by Haldane and Shastry for equidistantly localized spins on a cycle [1,2]. The Haldane-Shastry model extends the nearest-neighbor Heisenberg chain to long-range interactions, preserving the integrability. It can be considered also as a discrete analog of the integrable Calogero-Sutherland system describing particles confined on a cycle with a trigonometric inverse-square interaction [3]. The rational version of the latter had been proposed earlier and solved by Calogero [4]. A related discrete system was suggested and studied by Polychronakos and Frahm $[5,6]$. There are various integrable extensions of Calogero-type systems, in particular, to hyperbolic and elliptic potentials, particles with internal spin degrees of freedom (d.o.f.) [7], finite reflection groups, Coulomb potential [8], etc. (see Refs. [9,10] for a review).

Apart from the Liouville integrals [11], the unbound Calogero system (Calogero-Moser model) possesses additional integrals of motion ensuring maximal superintegralility [12-14]. This property is retained in the presence of the oscillator [15] and Coulomb potentials including the spaces with constant curvature [16].

There is an elegant way of solving and constructing integrals for quantum systems based on the Dunkl exchange operator [17]. The inverse-square (Calogero) interaction is hiding in the covariant derivative provided by such an operator $[18,19]$. The related connection is flat but includes the two-particle exchanges, making the constructed Hamiltonian, called sometimes a generalized Hamiltonian, and related observables essentially nonlocal. The unwanted exchanges disappear on the bosonic or

\footnotetext{
*tigran.hakobyan@ysu.am
}

Published by the American Physical Society under the terms of the Creative Commons Attribution 4.0 International license. Further distribution of this work must maintain attribution to the author(s) and the published article's title, journal citation, and DOI. Funded by SCOAP. fermionic states, recovering the original Calogero Hamiltonian. Likewise, the symmetries and wave functions of the generalized Hamiltonian are Dunkl-operator deformations of those for the underlying system without the inverse-square interaction. Once they are constructed, the integrals of motion of the original Calogero Hamiltonian are recovered as symmetric polynomials in symmetry generators of the generalized system.

In this context, the generalized Calogero Hamiltonian remains invariant with respect to the Dunkl-operator deformation of angular momentum generators. In addition, the hidden symmetry is provided by the Dunkl analog of the Fradkin tensor. Both of them form an extension of the conventional unitary symmetry of an isotropic oscillator [20] (see also a closely related description [21]). The hidden symmetry of the generalized Calogero-Moser system with a Coulomb potential is provided by a Dunkl deformed Runge-Lenz vector [16,22]. Recently, a Dunkl analog for the Dirac operator and its symmetries has been investigated [23].

At the equilibrium point corresponding to the potential minimum, the dynamical variables become frozen. The Hamiltonian reduces to the generalized PolychronakosFrahm chain with the coordinate exchanges instead of the spin ones. The application of the exchange operators to discrete systems has a long story. Using them, the commuting invariants for the Haldane-Shastry and PolychronakosFrahm spin chains have been constructed, proving the integrability $[5,24]$. The symmetry was expanded to the (non-Abelian) Yangian algebra $[25,26]$. Its generators may be obtained from the symmetry algebra of the parental Calogero-Sutherland and Calogero models. Because of the loss of continuous variables, this map eliminates a lot of symmetries. In particular, the quantum determinant of the Yangian algebra, which combines the commuting integrals containing the Hamiltonian, become a trivial constant in the freezing limit. Instead, the power-series expansion in Plank's constant was applied to the quantum determinant. Then, the first-order term provides the discrete models with another set of Liouville integrals [27,28]. This fact suggests 
that the conservation laws for the integrable chains with inverse-square interactions have more elaborate structure than those for their dynamical counterparts and are needed for further study.

In this article, we describe the symmetry of the $N$-site generalized Polychronakos-Frahm chain stressing out on its relation to the $U(N)$ symmetry of the isotropic oscillator, which is responsible for the maximal superintegrability. We start from the Dunkl-operator deformation of the unitary group generators, which provides the dynamical and hidden symmetries for the generalized Calogero model. At the lowest potential values, the dynamical d.o.f. become limited to the discrete jumps between degenerate minima giving rise to a chain model. We continue by extracting the conserving quantities for the discrete system from the deformed unitary generators, wherein the diagonal elements correspond to the previously constructed integrals. Algebraic relations between the reduced generators are derived also. The symmetric combinations of the invariants produce integrals of the Polychronakos-Frahm chain. We finish by inspecting the Coulomb confining potential in this context. The analog of the Runge-Lenz vector vanishes at the discrete level and does not lead to a conservation law.

\section{GENERALIZED CALOGERO MODEL}

The Calogero model is an integrable system of onedimensional identical particles interacting by an inversesquare potential and bound by an external harmonic force. It is given by the Hamiltionain [4],

$$
\hat{H}_{\mathrm{C}}=\frac{1}{2} \sum_{i=1}^{N}\left(\hat{p}_{i}^{2}+x_{i}^{2}\right)+\sum_{i<j} \frac{g(g \mp \hbar)}{x_{i j}^{2}} .
$$

Here, $\hat{p}_{i}=-\imath \hbar \partial_{i}$ is the momentum operator, $g$ is a coupling constant, and the $-(+)$ sign in the potential corresponds to the bosons (fermions). We use a conventional notation for the particle distance, $x_{i j}=x_{i}-x_{j}$. The oscillator frequency is set to unity.

Most properties of the Calogero model (and its various extensions), like (super)integrability, spectrum, wave functions, and conservation laws are conditioned by its slightly modified version known as a generalized Calogero Hamiltonian [18,19],

$$
\hat{H}=\frac{1}{2} \sum_{i=1}^{N}\left(\hat{p}_{i}^{2}+x_{i}^{2}\right)+\sum_{i<j} \frac{g\left(g-\hbar M_{i j}\right)}{x_{i j}^{2}} .
$$

It includes a permutation operator $M_{i j}$, which exchanges the coordinates of $i$ th and $j$ th particles. At a first glance, such a modification may look rather strange due to its nonlocality but for the identical particles it just reduces to the standard Calogero Hamiltonian (1). The advantage of the above Hamiltonian is the representation in terms of the deformed momentum operator with a derivative replaced with the Dunkl operator,

$$
\begin{aligned}
& \hat{H}=\frac{1}{2} \sum_{i=1}^{N}\left(\hat{\pi}_{i}^{2}+x_{i}^{2}\right), \\
& \hat{\pi}_{i}=\hat{p}_{i}+\sum_{j \neq i} \frac{l g}{x_{i j}} M_{i j} .
\end{aligned}
$$

The latter can be considered as a kind of flat nonlocal covariant derivative with the following algebra [17]:

$$
\begin{aligned}
& {\left[\hat{\pi}_{i}, \hat{\pi}_{j}\right]=\left[x_{i}, x_{j}\right]=0, \quad\left[x_{i}, \hat{\pi}_{j}\right]=i \hat{S}_{i j},} \\
& \hat{S}_{i j}=\left(\delta_{i j}-1\right) g M_{i j}+\delta_{i j}\left(\hbar+g \sum_{k \neq i} M_{i k}\right) .
\end{aligned}
$$

At the $g \rightarrow 0$ limit when the Calogero inverse-square potential is absent, the operators $\hat{\pi}_{i}, \hat{S}_{i j}$ are mapped to the $\hat{p}_{i}$ and $\hbar \delta_{i j}$, correspondingly, and the above relations are reduced to the Heisenberg algebra commutation rules.

A Dunkl-operator analog of lowering-rising operators is defined in the standard way $[7,18,19]$,

$$
\begin{gathered}
\hat{a}_{i}^{ \pm}=\frac{x_{i} \mp l \hat{\pi}_{i}}{\sqrt{2}} \\
{\left[\hat{a}_{i}, \hat{a}_{j}\right]=\left[\hat{a}_{i}^{+}, \hat{a}_{j}^{+}\right]=0, \quad\left[\hat{a}_{i}, \hat{a}_{j}^{+}\right]=\hat{S}_{i j} .}
\end{gathered}
$$

The generalized Hamiltonian can be expressed in terms of them,

$$
\hat{H}=\frac{1}{2} \sum_{i}\left(\hat{a}_{i}^{+} \hat{a}_{i}+\hat{a}_{i} \hat{a}_{i}^{+}\right)=\sum_{i} \hat{a}_{i}^{+} \hat{a}_{i}+\frac{\hbar N}{2}-S .
$$

Here, by $S$, the rescaled invariant of the permutation group algebra is denoted

$$
S=-g \sum_{i<j} M_{i j}, \quad\left[S, M_{i j}\right]=0 .
$$

As a result, the lowering-rising operators obey a standard spectrum generating relations $[7,19]$,

$$
\left[\hat{H}, \hat{a}_{i}^{ \pm}\right]= \pm \hbar \hat{a}_{i}^{ \pm} .
$$

So, any bilinear combination,

$$
\hat{E}_{i j}=\hat{a}_{i}^{+} \hat{a}_{j},
$$

satisfies the conservation law,

$$
\left[\hat{H}, \hat{E}_{i j}\right]=0 .
$$

The elements $\hat{E}_{i j}$ are Dunkl-operator deformations of the unitary group generators. Together with permutations $M_{i j}$, they provide entire algebra of symmetries for the 
generalized Calogero model. In addition, the following quadratic relation takes place among $\hat{E}_{i j}$ and $\hat{S}_{i j}$ [20]:

$$
\hat{E}_{i j}\left(\hat{E}_{k l}+\hat{S}_{k l}\right)=\hat{E}_{i l}\left(\hat{E}_{k j}+\hat{S}_{k j}\right) .
$$

The latter implies, in particular, the following commutation relation:

$$
\left[\hat{E}_{i j}, \hat{E}_{k l}+\hat{S}_{k l}\right]=\hat{E}_{i l} \hat{S}_{k j}-\hat{S}_{i l} \hat{E}_{k j}
$$

As a consequence, the diagonal elements are closed under commutation. Unlike the Cartan algebra, they are not Abelian but obey a simple commutation,

$$
\left[\hat{E}_{i i}, \hat{E}_{k k}\right]=\left(\hat{E}_{i i}-\hat{E}_{k k}\right) \hat{S}_{i k} .
$$

The above algebra ensures that the power sums form a system of Liouville integrals of the Calogero system [18],

$$
\hat{\mathcal{E}}_{k}=\sum_{i} \hat{E}_{i i}^{k}, \quad\left[\hat{\mathcal{E}}_{k}, \hat{\mathcal{E}}_{l}\right]=0 .
$$

The generalized Hamiltonian itself is expressed in terms of the first member in this family,

$$
\hat{H}=\hat{\mathcal{E}}_{1}-S+\frac{N \hbar}{2} .
$$

Moreover, it is a unique Casimir element (up to a nonessential constant term) of the Dunkl-deformed $u(N)$ algebra [20].

Remember that the same algebra (16) describes also the symmetries of the generalized Hamiltonian related to the Sutherland model [18], an analog of the Calogero-Moser system with trigonometric interactions [3].

The antisymmetric combinations of $\hat{E}_{i j}$ yield the Dunkl angular momentum components [13,29],

$$
\hat{L}_{i j}=\hat{E}_{i j}-\hat{E}_{j i}=x_{i} \hat{\pi}_{j}-x_{j} \hat{\pi}_{i} .
$$

Together with permutations, they produce a deformation of so $(N)$ algebra with a unique Casimir element given by the Dunkl angular momentum square $\hat{L}^{2}=\sum_{i<j} \hat{L}_{i j}^{2}$ shifted by a permutation invariant term [20],

$$
\hat{\mathcal{L}}_{2}=\hat{L}^{2}+S^{2}-\hbar(N-2) S, \quad\left[\hat{L}_{i j}, \hat{\mathcal{L}}_{2}\right]=0 .
$$

It can be considered as a generalized angular Calogero Hamiltonian, which is reduced to the angular part of the Calogero model for identical particles [30],
$\hat{H}=-\frac{\hbar^{2}}{2}\left(\partial_{r}^{2}+\frac{N-1}{r} \partial_{r}\right)+\frac{r^{2}}{2}+\frac{\hat{\mathcal{L}}_{2}}{2 r^{2}} \quad$ with $\quad r=\sqrt{x^{2}}$

The symmetric combinations of the deformed $u(N)$ generators produce a Dunkl-operator deformation for the well-known Fradkin tensor [31],

$$
\hat{I}_{i j}=\hat{E}_{i j}+\hat{E}_{j i}-\hat{S}_{i j}=\hat{\pi}_{i} \hat{\pi}_{j}+x_{i} x_{j} .
$$

Remember that the angular momentum and Fradkin tensor describe, respectively, the dynamical and hidden symmetries of the $N$-dimensional isotropic oscillator [32].

The diagonal algebra (16) has an Abelian basis obtained by applying a shift to its elements. The shift is a tail composed of exchange operators [26],

$$
\hat{D}_{i}=\hat{E}_{i i}-\hat{S}_{i}, \quad \hat{S}_{i}=\sum_{j=1}^{i-1} \hat{S}_{i j},
$$

where $\hat{S}_{1}=0$ is supposed. Together with permutations, the elements $\hat{D}_{i}$ satisfy the defining relations of degenerate affine Hecke algebra,

$\left[\hat{D}_{i}, \hat{D}_{j}\right]=0, \quad\left[\hat{D}_{k}, \hat{S}_{j j+1}\right]=0 \quad$ if $k \neq i, i+1$,

$\hat{D}_{i+1} \hat{S}_{j j+1}-\hat{S}_{j j+1} \hat{D}_{i}=g^{2}$.

Note that the tails $S_{i}$ satisfy the same relations; i.e., the above equations remain true upon the substitution $\hat{D}_{i} \rightarrow \hat{S}_{i}$. As a result, the modified diagonal elements can be considered as an analog of Liouville integrals for the generalized Hamiltonian (2), which may be expressed via them using the representation (9),

$$
\hat{H}=\sum_{i} \hat{D}_{i}+\frac{\hbar N}{2}
$$

The higher-order power sums define the higher Hamiltonians,

$$
\hat{\mathcal{D}}_{k}=\sum_{i} \hat{D}_{i}^{k}
$$

The second member, $\hat{\mathcal{D}}_{2}$, corresponds to the generalized Calogero-Sutherland model. Note that in contrast to the previous integrals (17), the permutation invariance is not evident but can be verified. More familiar are the monomials given by the generating function $\prod_{i}\left(u-\hat{D}_{i}\right)$ [26]. In general, any symmetric polynomial in $\hat{\mathcal{D}}_{i}$ is permutation invariant and reduced to the constant of motion of the Calogero model (1) for indistinguishable particles.

More symmetric polynomial may include the nondiagonal components of the generators $\hat{E}_{i j}$. The permutations 
can be used in such expressions as well. Here are some simplest examples of such integrals,

$\sum_{i, j} \hat{I}_{i j}^{k}, \quad \sum_{i, j} \hat{L}_{i j}^{2 k}, \quad \sum_{i<j} \hat{I}_{i j}^{k} M_{i j}, \quad \sum_{i, j} \hat{I}_{i i}^{k} \hat{L}_{i j}^{2 l}$.

\section{GENERALIZED POLYCHRONAKOS-FRAHM CHAIN}

Let us set the interaction constant to unity, $g=1$. In the current section, we consider the generalized Calogero model at the equilibrium point, where the (classical) confining Calogero potential takes its minimal value,

$$
\frac{\partial V}{\partial x_{i}}=0, \quad V(x)=\sum_{i=1}^{N} \frac{x_{i}^{2}}{2}+\sum_{i<j} \frac{1}{x_{i j}^{2}} .
$$

The coordinates are given by the roots of the $N$ th-order Hermite polynomial [6]. All roots differ, so there are $N$ ! equivalent minima connecting by the coordinate exchanges $M_{i j}$. These are only allowed evolutions in the frozen system.

Consider the expansion of the Hamiltonian (2) in powers of Plancks constant,

$$
\hat{H}=V+\hbar H^{(1)}-\frac{\hbar^{2} \partial^{2}}{2} .
$$

The first-order term can be considered as a generalized Polychronakos-Frahm Hamiltonian, where the spin exchange operator is replaced by the coordinate exchange [5],

$$
H^{(1)}=\sum_{i<j} \frac{1}{x_{i j}^{2}} M_{i j} .
$$

The $S U(n)$ symmetric spin chain is recovered from the above Hamiltonian after the replacement of the coordinate permutations with spin exchange operators so that

$$
H_{\mathrm{PF}}=\sum_{i<j} \frac{P_{i j}}{x_{i j}^{2}} .
$$

Here, $P_{i j}$ permutes the $i$ th and $j$ th spins, which take values in the fundamental representation of the $S U(n)$ group. Both Hamiltonians become identical on the bosonic (fermionic) states provided that the particles are endowed with additional spin d.o.f. Then the entire wave function must be symmetric (antisymmetric) under simultaneous exchanges of coordinates and spins for bosons (fermions). A permutation of spatial coordinates $M_{i j}$ can be replaced by the spin exchange operators $P_{i j}$ and $-P_{i j}$ in the bosonic and fermionic cases, respectively. Note that the projection inverts the order of permutations so that the operator $M_{i j} M_{k l}$ must be substituted by $P_{k l} P_{i j}$.
Let us now construct the constants of motion of the chain Hamiltonian (30) by applying the $\hbar$ expansion to the integrals of the original dynamical model (2) at the equilibrium point. They do not provide directly the invariants of the $S U(n)$ spin chain (31). In order to get them, one needs to carry out symmetrization over all particles prior to the projection. For instance, $H^{(1)}$ stays invariant under a selected coordinate exchange, $M_{i j}$, but $H_{\mathrm{PF}}$ does not preserve its spin counterpart, $P_{i j}$. However, both Hamiltonians preserve the symmetrized version given by the element $S$ (10).

The Dunkl momentum (4) and permutation matrix (6) can be presented as follows:

$$
\begin{array}{r}
\hat{\pi}_{i}=\pi_{i}-\imath \hbar \partial_{i} \quad \text { with } \quad \pi_{i}=\sum_{j \neq i} \frac{l}{x_{i j}} M_{i j}, \\
\hat{S}_{i j}=S_{i j}+\hbar \delta_{i j} \quad \text { with } \quad S_{i j}=\left(\delta_{i j}-1\right) M_{i j}+\delta_{i j} \sum_{k \neq i} M_{i k} .
\end{array}
$$

Here and in the following, the superscript is omitted in the zeroth-order term of any operator, so that $\pi_{i}^{0}=\pi_{i}$. In that limit, the canonical commutation relations resemble their original form (5),

$\left[\pi_{i}, \pi_{j}\right]=0, \quad\left[x_{i}, \pi_{j}\right]=\imath S_{i j}, \quad S_{i j}= \begin{cases}-M_{i j}, & \text { if } i \neq j, \\ \sum_{k \neq i} M_{i k}, & \text { otherwise } .\end{cases}$

Recall now that the particle coordinates are set by the roots of the Hermite polynomial, which imposes certain algebraic relations on them (see, for example, [27,33]). As a result, the discrete Dunkl momenta are not independent any more but undergo additional algebraic constraints. In particular, the following relations hold among the fixed phase space variables:

$$
\sum_{i} x_{i}=\sum_{i} \pi_{i}=\sum_{i} S_{i k}=0,
$$

$x^{2}=\pi^{2}=\sum_{i<j} \frac{2}{x_{i j}^{2}}=\frac{1}{2} N(N-1), \quad x \cdot \pi=-\pi \cdot x=-l S$.

In general, all relations between the operators of the dynamical Calogero system are preserved at the $\hbar=0$ limit. In particular, the frozen lowering-rising operators

$$
a_{i}^{ \pm}=\frac{x_{i} \mp \imath \pi_{i}}{\sqrt{2}}, \quad \hat{a}_{i}^{ \pm}=a_{i}^{ \pm} \pm \frac{\hbar}{\sqrt{2}} \partial_{i}
$$

obey a rule similar to the commutations of the deformed Heisenberg algebra (8) [5], 


$$
\left[a_{i}, a_{j}\right]=\left[a_{i}^{+}, a_{j}^{+}\right]=0, \quad\left[a_{i}, a_{j}^{+}\right]=S_{i j} .
$$

Because of the minimum condition (28), the spectrum generating relation (11) remains valid for the generalized Polychronakos-Frahm chain too [5],

$$
\left[H^{(1)}, a_{i}^{ \pm}\right]= \pm a_{i}^{ \pm} .
$$

However, unlike the dynamical case, the discrete Hamiltonian is not expressed via lowering-rising operators [see Eq. (9)].

The constants of motion of the generalized Calogero model (12) have terms up to the second order in their expansion,

$$
\hat{E}_{i j}=E_{i j}+\hbar E_{i j}^{(1)}-\frac{\hbar^{2}}{2} \partial_{i} \partial_{j}
$$

The relations (39) imply conservation of the constant terms,

$$
E_{i j}=a_{i}^{+} a_{j}, \quad\left[H^{(1)}, E_{i j}\right]=0 .
$$

For the Dunkl angular momentum (19), the $\hbar^{2}$ part vanishes, while the $\hbar$ term corresponds to the usual angular momentum operator in quantum mechanics,

$\hat{L}_{i j}=L_{i j}-\imath \hbar\left(x_{i} \partial_{j}-x_{j} \partial_{i}\right), \quad L_{i j}=x_{i} \pi_{j}-x_{j} \pi_{i}$.

A similar expansion for the Fradkin tensor is more complex,

$\hat{I}_{i j}=I_{i j}+\hbar I_{i j}^{(1)}-\hbar^{2} \partial_{i} \partial_{j}, \quad I_{i j}=x_{i} x_{j}+\pi_{i} \pi_{j}$.

The first order operator-valued coefficient is given by

$$
\begin{gathered}
I_{i j}^{(1)}=\frac{1}{x_{i j}^{2}} M_{i j}+\sum_{k \neq i, j}\left(\frac{\partial_{i}}{x_{j k}} M_{j k}+\frac{\partial_{j}}{x_{i k}} M_{i k}\right) \text { for } i \neq j, \\
I_{i i}^{(1)}=\sum_{k \neq i} \frac{1}{x_{i k}}\left(\partial_{i}+\partial_{k}-\frac{1}{x_{i k}}\right) M_{i k} .
\end{gathered}
$$

As was discussed above, the algebraic relations between the symmetry generators of the dynamical system remain true at the freezing limit. In particular, the most general relation (14) and its consequences (15), (16) are reduced, respectively, to the following equations:

$$
\begin{gathered}
E_{i j}\left(E_{k l}+S_{k l}\right)=E_{i l}\left(E_{k j}+S_{k j}\right), \\
{\left[E_{i j}, E_{k l}+S_{k l}\right]=E_{i l} S_{k j}-S_{i l} E_{k j},} \\
{\left[E_{i}, E_{k}\right]=\left(E_{i}-E_{k}\right) S_{i k} .}
\end{gathered}
$$

The power sums of diagonal elements yield Liouville integrals of the Polychronakos-Frahm chain [5],

$\mathcal{E}_{k}=\sum_{i} E_{i i}^{k}, \quad\left[\mathcal{E}_{k}, \mathcal{E}_{l}\right]=0, \quad\left[H^{(1)}, \mathcal{E}_{k}\right]=0$.

The first element of this set is rather trivial, $\mathcal{E}_{1}=S+\frac{N(N-1)}{2}$, as is easy to get using the Eqs. (36) and (37). The higher rank $\mathcal{E}_{k}$ have more complicated expressions.

For the dynamical system, the quadratic relations (14) are the only constraints which the symmetry generators $\hat{E}_{i j}$ obey [20]. However, there are a lot of other restrictions on them at the equilibrium point. For example, the Eqs. (35) imply the sum vanishing rules,

$$
\sum_{i} E_{i k}=\sum_{i} E_{k i}=\sum_{i} L_{i k}=\sum_{i} I_{i k}=0 .
$$

In the dynamical case, the angular Calogero Hamiltonian (20) plays an important role among constants of motion. In the absence of an oscillator potential, it maps the Liouville set to additional integrals. However, in the equilibrium limit, the angular part does not produce a new integral but just is expressed via trivial ones. Using the relations (35), (36), (19), and (51), it is easy to verify that the operator $\hat{\mathcal{L}}_{2}$ is a scalar at the equilibrium. Its $\hbar$-linear coefficient reproduces the chain Hamiltonian as was argued earlier [30],

$$
\mathcal{L}_{2}=r^{4}, \quad \mathcal{L}_{2}^{(1)}=2 r^{2} H^{(1)}
$$

with $r^{2}=\frac{1}{2} N(N-1)$ (36).

Let us calculate also a chain analog of the Fradkin's tensor square. It corresponds to the second $(k=2)$ member of the first sequence presented in (27),

$\sum_{i, j} I_{i j}^{2}=\sum_{i \neq j \neq k} M_{i j k}-2 S^{2}+\frac{1}{2} N(N-1)\left(N^{2}-N+4\right)$.

Here, $M_{i j k}$ is a cyclic permutation of the marked three coordinates. It also is expressed through the invariants of the permutation group algebra. Nevertheless, we expect that higher degree power sums from (27) at the equilibrium give rise to nontrivial integrals of motion for the chain Hamiltonians (30), (31).

Finally, consider the shifted diagonal elements (23). At the freezing level, they also commute

$$
\left[D_{i}, D_{j}\right]=0, \quad D_{i}=E_{i i}-S_{i} .
$$

Together with permutations, they form also degenerate affine Hecke algebra (24) (with $g=1$ ). Contrary to the nonshifted case (41), (49), the related symmetric polynomials, $\mathcal{D}_{k}=\sum_{i} D_{i}^{k}$, are scalars (multiples of identity) and do not lead to a conservation law. At the same time, the $\hbar$-linear terms of the dynamical integrals (26) form a family of commuting nontrivial integrals (49), 
$\mathcal{D}_{k}^{(1)}=\sum_{i} \sum_{l=0}^{k-1} D_{i}^{l} D_{i}^{(1)} D_{i}^{k-l-1}, \quad\left[\mathcal{D}_{k}^{(1)}, \mathcal{D}_{l}^{(1)}\right]=0$,

where $D_{i}^{(1)}=\frac{1}{2} I_{i i}^{(1)}$. The first element of the family describes the chain Hamiltonian: $\mathcal{D}_{1}^{(1)}=-2 H^{(1)}$. This remarkable property was established first for the Haldane-Shastry chain using the Yangian represtation [28] and has been extended later to the PolychonakosFrahm chain [27].

\section{RELATION TO GENERALIZED CALOGERO-COULOMB SYSTEM}

One can start from another radial confining potential in the dynamical system (2) in order to get its discrete analog at the semiclassical limit (30). Apart from the oscillator case, the Coulomb potential is preferable among others since it leads to a superintegrable system as well [16],

$$
\hat{H}_{\text {Coul }}=\sum_{i=1}^{N} \frac{\hat{\pi}_{i}^{2}}{2}-\frac{1}{r} .
$$

So, there is a hope to extract more integrals for the chain system as a reduction of the recently obtained Dunkloperator analog of the Runge-Lenz vector [22],

$$
\hat{A}_{i}=\frac{1}{2} \sum_{j}\left\{\hat{L}_{i j}, \hat{\pi}_{j}\right\}+\frac{l}{2}\left[\hat{\pi}_{i}, \hat{S}\right]-\frac{x_{i}}{r} .
$$

Together with the Dunkl angular momentum $\hat{L}_{i j}$, it yields the Dunkl-operator deformation of the $S O(N+1)$ group generators, which describe the symmetry of the generalized Calogero system with Coulomb potential (55) [22].

The equilibrium point is defined now by a solution of the equations,

$\frac{\partial V_{\text {Coul }}}{\partial y_{i}}=0, \quad V_{\text {Coul }}(y)=-\frac{1}{r_{y}}+\sum_{i<j} \frac{1}{y_{i j}^{2}}, \quad r_{y}^{2}=x^{2}$.

In order to separate it from the oscillator minimum (28), the Coulomb point is marked by $y$. It can be obtained from the oscillator point, given by zeros of Hermite polynomial (28), by the map $y_{i}=r^{3} x_{i}$. The latter implies that $r_{y}=r^{4}$. Therefore, passing from the oscillator to Coulomb potential, the chain Hamiltonian (30) just undergoes a rescaling $H_{\text {Coul }}^{(1)}=r^{-6} H^{(1)}$. Evidently, the Dunkl momentum transforms as $r^{-3} \pi_{i} \rightarrow \pi_{i}$. The relations (35), (36) stay unchanged apart from the first equation in (36), which must be replaced now by

$$
\frac{1}{r_{y}}=\pi^{2}=\sum_{i<j} \frac{2}{y_{i j}^{2}} .
$$

Using them, it is easy to verify that the Dunkl deformed Runge-Lenz vector vanishes trivially at the Coulomb equilibrium point,

$$
A_{i}=\frac{1}{2} \sum_{j}\left\{L_{i j}, \pi_{j}\right\}+\frac{l}{2}\left[\pi_{i}, S\right]-\frac{y_{i}}{r_{y}}=0 .
$$

This fact eliminates the power sums $\hat{\mathcal{A}}_{k}=\sum_{i} \hat{A}_{i}^{k}$ at the equilibrium, $\mathcal{A}_{k}=0$, but does not necessarily imply the vanishing of the first-order operator in powers of $\hbar$. Actually, the first two such operators are

$$
\mathcal{A}_{1}^{(1)}=0, \quad \mathcal{A}_{2}^{(1)}=S / r_{y},
$$

as can be derived from the definition (56). Note that the square of the Dunkl Runge-Lenz vector may be shifted certain way to become compatible with the Dunkl angular momentum. Moreover, it provides a deformation of the well-known relation between the angular momentum and Runge-Lenz squares [22],

$$
\begin{aligned}
& \hat{\mathcal{A}}_{2}^{\prime}=\hat{\mathcal{A}}_{2}+2 \hbar S \hat{H}_{\text {Coul }}, \quad\left[\hat{L}_{i j}, \hat{\mathcal{A}}_{2}\right]=0, \\
& \hat{\mathcal{A}}_{2}^{\prime}=2 \hat{H}_{\text {Coul }} \hat{\mathcal{L}}_{2}+\frac{\hbar^{2}(N-1)^{2}}{2} \hat{H}_{\text {Coul }}+1 .
\end{aligned}
$$

Applying the Taylor expansion with the coefficients written in terms of $y$ coordinates,

$$
\mathcal{L}_{2}=r_{y}, \quad \mathcal{L}_{2}^{(1)}=2 r_{y}^{2} H_{\text {Coul }}^{(1)}, \quad V_{\text {Coul }}=-\frac{1}{2 r_{y}},
$$

we obtain the vanishing condition, $\mathcal{A}_{2}^{\prime(1)}=0$, which is equivalent to the second equation in (60).

\section{ACKNOWLEDGMENTS}

The author is grateful to Armen Nersessian for stimulating discussions. This work was supported by the Armenian State Committee of Science Grants No. SFU02, No. 18RF-002, and No. 18T-1C106. It was fulfilled within the ICTP Affiliated Center Program AF-04. 
[1] F. D. M. Haldane, Exact Jastrow-Gutzwiller ResonatingValence-Bond Ground State of the Spin-1/2 Antiferromagnetic Heisenberg Chain with $1 / r^{2}$ Exchange, Phys. Rev. Lett. 60, 635 (1988).

[2] B.S. Shastry, Exact Solution of an $S=1 / 2$ Heisenberg Antiferromagnetic Chain with Long-Ranged Interactions, Phys. Rev. Lett. 60, 639 (1988).

[3] B. Sutherland, Exact results for a quantum many-body problem in one dimension, Phys. Rev. A 4, 2019 (1971); Exact results for a quantum many-body problem in one dimension. II, Phys. Rev. A 5, 1372 (1972).

[4] F. Calogero, Solution of a three-body problem in one dimension, J. Math. Phys. (N.Y.) 10, 2191 (1969); Solution of the one-dimensional $N$-body problems with quadratic and/or inversely quadratic pair potentials, J. Math. Phys. (N.Y.) 12, 419 (1971).

[5] A. Polychronakos, Lattice Integrable Systems of HaldaneShastry Type, Phys. Rev. Lett. 70, 2329 (1993).

[6] H. Frahm, Spectrum of a spin chain with inverse square exchange, J. Phys. A: Math. Gen. 26, L473 (1993).

[7] J. A. Minahan and A. P. Polychronakos, Integrable systems for particles with internal degrees of freedom, Phys. Lett. B 302, 265 (1993).

[8] A. Khare, Exact solution of an $N$-body problem in one dimension, J. Phys. A 29, L45 (1996).

[9] M. A. Olshanetsky and A. M. Perelomov, Classical integrable finite dimensional systems related to Lie algebras, Phys. Rep. 71, 313 (1981); Quantum integrable systems related to Lie algebras, Phys. Rep. 94, 313 (1983).

[10] A. P. Polychronakos, Physics and mathematics of Calogero particles, J. Phys. A 39, 12793 (2006).

[11] J. Moser, Three integrable Hamiltonian systems connected with isospectral deformations, Adv. Math. 16, 197 (1975).

[12] S. Wojciechowski, Superintegrability of the CalogeroMoser system, Phys. Lett. A 95, 279 (1983).

[13] V. B. Kuznetsov, Hidden symmetry of the quantum Calogero-Moser system, Phys. Lett. A 218, 212 (1996).

[14] C. Gonera, A note on superintegrability of the quantum Calogero model, Phys. Lett. A 237, 365 (1998).

[15] C. Gonera and P. Kosinski, Calogero model and sl(2,R) algebra, Acta Phys. Pol. B 30, 907 (1999).

[16] T. Hakobyan, O. Lechtenfeld, and A. Nersessian, Superintegrability of generalized Calogero models with oscillator or Coulomb potential, Phys. Rev. D 90, 101701(R) (2014).

[17] C. F. Dunkl, Differential-difference operators associated to reflection groups, Trans. Am. Math. Soc. 311, 167 (1989).

[18] A. Polychronakos, Exchange Operator Formalism for Integrable Systems of Particles, Phys. Rev. Lett. 69, 703 (1992).
[19] L. Brink, T. Hansson, and M. Vasiliev, Explicit solution to the N-body Calogero problem, Phys. Lett. B 286, 109 (1992).

[20] M. Feigin and T. Hakobyan, On the algebra of Dunkl angular momentum operators, J. High Energy Phys. 11 (2015) 107.

[21] A. Turbiner, Hidden algebra of the $N$-body Calogero problem, Phys. Lett. B 320, 281 (1994).

[22] T. Hakobyan and A. Nersessian, Runge-Lenz vector in Calogero-Coulomb problem, Phys. Rev. A 92, 022111 (2015).

[23] H. De Bie, R. Oste, and J. Van der Jeugt, On the algebra of symmetries of Laplace and Dirac operators, Lett. Math. Phys. 108, 1905 (2018); The total angular momentum algebra related to the $S_{3}$ Dunkl Dirac equation, Ann. Phys. (Amsterdam) 389, 192 (2018).

[24] M. Fowler and J. A. Minahan, Invariants of the HaldaneShastry $S U(N)$ Chain, Phys. Rev. Lett. 70, 2325 (1993).

[25] F. D. M. Haldane, Z. N. C. Ha, J. C. Talstra, D. Bernard, and V. Pasquier, Yangian Symmetry of Integrable Quantum Chains with Long-Range Interactions and a New Description of States in Conformal Field Theory, Phys. Rev. Lett. 69, 2021 (1992).

[26] D. Bernard, M. Gaudin, F. D. M. Haldane, and V. Pasquier, Yang-Baxter equation in spin chains with long range interactions, J. Phys. A 26, 5219 (1993).

[27] P. Mathieu and Y. Xudous, Conserved charges of nonYangian type for the Frahm-Polychronakos spin chain, J. Phys. A 34, 4197 (2001).

[28] J. C. Talstra and F. D. M. Haldane, Integrals of motion of the Haldane Shastry Model, J. Phys. A 28, 2369 (1995).

[29] M. Feigin, Intertwining relations for the spherical parts of generalized calogero operators, Theor. Math. Phys. 135, 497 (2003).

[30] M. Feigin, O. Lechtenfeld, and A. Polychronakos, The quantum angular Calogero-Moser model, J. High Energy Phys. 07 (2013) 162.

[31] F. Correa, T. Hakobyan, O. Lechtenfeld, and A. Nersessian, Spherical Calogero model with oscillator/Coulomb potential: Quantum case, Phys. Rev. D 93, 125009 (2016).

[32] D. M. Fradkin, Existence of the dynamic symmetries $O_{4}$ and $\mathrm{SU}_{3}$ for all classical central potential problems, Theor. Phys. 37, 798 (1967); Three-dimensional isotropic harmonic oscillator and $S U_{3}$, Am. J. Phys. 33, 207 (1965).

[33] F. Calogero, Equilibrium configuration of the onedimensional $n$-Body problem with quadratic and inversely quadratic pair potentials, Lett. Nuvo Cimento 20, 251 (1977). 